\title{
CAPITAL SOCIAL: IMPORTANCIA DE LAS MEDICIONES PARA COLOMBIA
}

\section{SOCIAL CAPITAL: IMPORTANCE OF MEASEREMENTS FOR COLOMBIA}

\author{
Julio Foliaco-Gamboa ${ }^{1}$
}

Forma de citar: FOLIACO-GAMBOA Julio. Capital social: Importancia de las mediciones para Colombia. Respuestas. 2013; 18(2): 43-60.

Recibido:

Abril 2 de 2013

Aceptado:

Septiembre 16 de 2013

\footnotetext{
${ }^{1}$ Magister en Administración Universidad Francisco de Paula Santander jefoliacog@unal.edu.co Cúcuta - Colombia
}

\section{RESUMEN:}

El capital social como nuevo paradigma de investigación contribuye a encontrar respuestas a las profundas diferencias del desarrollo social y económico. Instituciones internacionales y países han intensificado en estas investigaciones desde la década de los ochenta. Los propósitos específicos son: 1. Analizar el concepto del capital social como nuevo paradigma universal de investigación; 2. Ponderar los resultados de las mediciones e investigaciones hechas en Colombia con el liderazgo del profesor John Sudarsky desde 1997; 3. Comparar las mediciones nacionales de capital social con las mediciones de otros países y regiones; y 4. Establecer las causas estructurales del bajo capital social colombiano y hacer reflexiones para su mejoramiento.

Palabras clave: articulación social, confianza institucional, capital humano, capital social, desarrollo económico, sociedad civil.

\section{ABSTRACT:}

The social capital as new research paradigm contributes to find out answers to big differences between social and economic development. International institutions and countries have intensified on these researches since the eighty's decade. The specific purposes are: 1. Analyzing the concept of social capital as a new and universal research paradigm; 2. Enhance the results of measurements and research made in Colombia with Professor John Sudarsky's leadership since 1997; 3. Compare the national measurements of social capital with those of other countries and regions; 4. Establish the structural reasons of the Colombian low social capital, making some reflections towards its improvement.

Keywords: social articulation, institutional trust, human capital, social capital, economic development, civil society. 


\section{INTRODUCCIÓN}

PP: 43-60

E capital social como conjunto de relaciones y sinergias entre ciudadanos e instituciones, es un concepto que se ha desarrollando a partir los escritos de Lyda J Hanifan (1916), un supervisor de colegios en Virginia del Oeste, quien relacionó el mejor desempeño escolar con la integración entre familiares y comunidad para gestar un "capital social" a favor de toda la sociedad (Woolcock, 1998). El mayor desarrollo de este concepto se ha dado gracias a los trabajos de Bourdieu, Coleman, Woolcock, Putman, y otros estudiosos del tema, desde la década de 1980. Los debates sobre el capital social se concentran en su conceptualización, relaciones entre comunidades e instituciones y mediciones e impactos en diferentes contextos sociales con la confluencia de la sociología y la economía.

Bourdieu (1985) define el capital social como un valor de recursos perteneciente a las relaciones entre ciudadanos y miembros de una sociedad más o menos institucionalizada. Esta teoría refuerza la expansión del capital en los ámbitos económico y sociológico. A su vez el autor presenta tres tipos de capitales: el económico relacionado con el dinero; el cultural relacionado con la formación y capacitación de los ciudadanos y también convertible en dinero a través de la relación educación y trabajo; y el capital social que relaciona a las personas con las instituciones y orienta al logro de objetivos socioeconómicos comunes.

Coleman (1988) se apoyó en la sociología y la economía para aplicar el concepto de capital social y demostrar que las acciones conjuntas de los individuos afectan el desarrollo de las organizaciones. Desarrolló, al igual que Bourdieu, tres tipos de capital: a) capital físico en su relación con la producción; b) capital humano en función de capacidades y habilidades de las personas; y c) capital social subdividido en tres formas: 1) obligaciones, expectativas y estructuras de confianza haciendo referencia al ámbito individual; 2) redes de información referido a la interacción del individuo y las instituciones; y 3) normas y sanciones efecti- vas con referencia al papel de las instituciones estatales y medios de información para la generación de condiciones de un mejor capital social.

Por su parte Woolcock (1998) trata de hacer converger los conceptos de capital social planteando que la familia, los amigos y las asociaciones constituyen un activo para utilizarlo en beneficio propio, superar una crisis u obtener beneficios económicos. Esta idea básica puede ampliarse a grupos y comunidades integradas por diversas organizaciones cívicas y sociales que tienen una posición más fuerte para enfrentar la pobreza y vulnerabilidad (Moser, 1996).

Putnam (1995), asocia el capital social con el desarrollo económico, en una relación funcional y con aplicación de los enfoques teóricos de Bourdieu, Coleman y Woolcock en diferentes países y regiones. De esta forma configura un nuevo paradigma del capital social en el campo de la investigación socioeconómico en las últimas tres décadas.

De las diferencias encontradas, Bourdieu vincula el análisis individual y las obligaciones del individuo con la sociedad, considerando que cualquier tipo de capital social es convertible a lo económico. Para Coleman, el análisis del capital social es multidimensional por las relaciones entre individuos, comunidades, organizaciones y el Estado. Por su parte, Putnam concentra su visión en la inter funcionalidad pragmática de los individuos, las organizaciones, el contexto y el Estado para mejorar el desarrollo. Woolcock considera que los individuos y los grupos comunitarios realizan en su interior la génesis principal de su desarrollo pero requieren ampliar sus relaciones con otros grupos para lograrlo.

Lucas (1988) dilucidó el papel del capital humano a través de tres modelos: el primero se concentra en la acumulación del capital físico y tecnológico; el segundo en la acumulación del capital humano a través de la educación; y el tercero, en un modelo especializado de capital humano como aprendizaje práctico. En esta misma se reflexiona sobre el capital hu- 
mano como modelo de crecimiento endógeno, donde se presentan dos cuestionamientos: ¿cuáles son las diferencias entre la educación, la experiencia - lo intangible - y la ciencia? ¿Cómo el conocimiento y la ciencia inciden en la producción? Estos interrogantes surgieron de las mediciones de la alfabetización, al entender que la educación incide en la inversión y contribuye a predecir futuras tasas de crecimiento. Entonces, es claro que el capital humano es un factor determinante del capital social, donde el primero es una acción individual y el segundo un conjunto de acciones colectivas.

Con referencia al desarrollo económico, en la década de 1980, las teorías económicas incorporaron el capital social como factor incidente en las tasas de crecimiento económico. Para Todaro (1985), el desarrollo económico es un proceso de incremento del ingreso per cápita de un país durante un largo plazo, siempre que la línea de pobreza absoluta no se incremente y la distribución del ingreso sea equitativa. Por consiguiente, el desarrollo económico debe interpretarse como un progreso económico, un incremento del bienestar de la población y una correlación entre la prosperidad de sector rural y la prosperidad del sector urbano. Igualmente, Meier (1984) había expresado que el incremento del ingreso per cápita no es condición suficiente sino una parte del bienestar económico.

Este marco conceptual ha sido un referente para las tres mediciones de capital social hechas en Colombia por medio del Barómetro de Capital Social (BARCAS), un instrumento validado mediante la aplicación de muestras nacionales que mide indicadores sociales relativos al desarrollo ciudadano, el desempeño institucional y la articulación de la sociedad civil y las instituciones del Estado (Sudarsky, 1999). Estas investigaciones arrojaron resultados sobre los aspectos que se deben fortalecer, como la acción colectiva, la democracia y el desempeño institucional.

Todo el trabajo con el BARCAS se ha enmarcado en la relación funcional entre capital social y desarrollo económico. Con metodo- logía, dimensiones y comparaciones nacionales e internacionales, incluyendo análisis de causalidad lineal, apoyado en la continuidad histórica de diez dimensiones y los valores encontrados en cada medición (1997, 2005 y 2011). Asimismo, se realiza un análisis de causalidad múltiple teniendo en cuenta el contexto, la educación, el tamaño de la población, los medios y la confianza institucional, entre otros factores. Finalmente, se registran otras mediciones como Barómetro de las Américas (2012), hechas por organismos nacionales e internacionales, para concluir este trabajo con aportes, importancia y avances de las mediciones del capital social en Colombia desde 1997.

\section{GÉNESIS DE LAS MEDICIONES DEL CAPITAL SOCIAL EN COLOMBIA}

Las mediciones hechas en Colombia están basadas en las investigaciones de Coleman (1990) y los trabajos de aplicación de Putman et al., (1995; 1994), cuyos instrumentos e indicadores han sido implementados en más de 78 países como Australia, Estados Unidos, Uganda, Ghana, Nueva Zelandia, Italia, Nigeria, entre otros (Inglehart, 2002). Las investigaciones realizadas en países del sudeste asiático son referentes de desarrollo económico asociado con articulación social y convivencia. Esta relación entre lo social y lo económico asumida en Colombia por Sudarsky está en la misma vía de lo propuesto por Putnam, Leonardi, y Nanetti (1994) y Coleman $(1988,1990)$.

La aplicación del Barcas en Colombia (1997, 2005 y 2011) tomó como referencia la Encuesta Mundial de Valores (EMV), ya probada en otros países y regiones del mundo. En nuestro país se adelantaron más de tres mil encuestas en cada medición, en diferentes regiones, con rigor estadístico, contribuyendo con el desarrollo del tema del capital social como paradigma universal de investigación.

En su trabajo, "La Evolución del Capital Social en Colombia 1997 - 2005”, John Sudarsky
Junio - Diciembre 2013 ISSN 0122-820X PP: $43-60$ 
No. 2

Junio - Diciembre 2013 ISSN 0122-820X

PP: 43-60
(2007) relaciona la participación ciudadana en "los asuntos de interés público" con el crecimiento económico en el mediano y largo plazo. Concretamente, las relaciones horizontales entre los miembros locales de la comunidad y las relaciones verticales entre éstos y las instituciones del Estado. El resultado de estas mediciones conduce a la necesidad de fortalecer la sociedad civil en su participación política, nivel educativo y confianza en el Estado. Asimismo, se dan explicaciones a la baja confianza interpersonal, la alta inequidad social, la brecha persistente entre lo urbano y lo rural, la baja credibilidad en los medios de comunicación, el atomismo sociopolítico, el alto clientelismo y la baja confianza ciudadana en las instituciones del Estado. Todas las mediciones en nuestro país registran bajo capital social caracterizado por baja articulación entre ciudadanos y entre éstos y las instituciones del Estado (Sudarsky, 2007).

En general, se encontraron respuestas implícitas y explícitas a las preguntas: ¿Por qué los colombianos no tenemos mayor interés por los asuntos públicos? ¿Por qué existe poca articulación social entre los colombianos? ¿Cuáles son las causas de los altos niveles de desconfianza? ¿Por qué un país rico en recursos naturales no tiene un desarrollo sostenible e inclusivo?

Las soluciones a estas preguntas difieren de las interpretaciones que estiman la baja productividad y los bajos niveles educativos como causas primarias de la inequidad social y la pobreza extrema, subestimando la convergencia de causas contextuales, estructurales y políticas con mayor impacto en el desarrollo.

Otros hallazgos hacen referencia a la disfuncionalidad de las entidades públicas, caracterizadas por prácticas como el corporativismo excluyente, el clientelismo y el particularismo. Estas disfunciones han generado una élite de dirigentes que utilizan su vinculación como servidores públicos para favorecer sus propios intereses, lo cual incrementa el aislamiento entre electores y elegidos con incidencia en el círculo vicioso del subdesarrollo: "los pobres son cada vez más pobres y en mayor cantidad".
Las trascendentales conclusiones de los BARCAS de 1997 y 2005 hechas por el profesor Sudarsky y la medición hecha con el mismo instrumento por el profesor Hurtado (2011) articulando las mediciones anteriores, se han convertido en los referentes de las mediciones de capital social en Colombia, validados por mediciones internacionales. Estos documentos a disposición de las instituciones del Estado se convierten en aportes para la ejecución de planes integradores entre la sociedad civil, las instituciones del Estado y los gremios económicos en procura de un desarrollo sostenible.

\section{RESULTADOS DE LAS MEDICIONES DEL CAPITAL SOCIAL - 1997 Y 2005}

El propósito de este aparte es analizar la metodología y los resultados de la segunda medición del capital social hecha en Colombia en 2005 y resaltar los cambios con referencia a la primera medición de 1997. La coherencia de los resultados reafirma la conveniencia de una mayor participación de la sociedad civil en la estructura de decisiones con comunidades, gobierno, gremios y grupos políticos, tal como lo establece la Constitución (Colombia, constitución política de 1991).

Para seleccionar las preguntas, medir dimensiones y validar el instrumento se aplicaron preguntas previas (cuatrocientos formularios) a formaciones sociales diversas. En la muestra definitiva se aplicaron criterios como tamaño del municipio, regionalización, distribución proporcional entre cabecera y el municipio, género y edad. En ciudades grandes como Bogotá, Medellín, Cali y Barrancabermeja se tomaron muestras de mil habitantes. En total se incluyeron en el estudio catorce regiones -departamentos y subregiones-y cincuenta y cuatro municipios. En las comunas se aplicaron mil encuestas (Sudarsky, 2007).

En la metodología empleada resulta novedosa la inclusión de dos factores diferentes al capital social (Ksocial), identificados por el autor como Fuentes de Información no Validadas 
(Fenoval) y Confianza en Fuentes de Información del Estado (Confie). Fenoval establece cuando una persona tiene fe en una información, sin que recurra a su comprobación. Es una fe producto de una construcción social a partir de racionalidades propias. Con este nuevo factor se puede medir el efecto de variables exógenas como educación, ubicación urbana o rural, el estrato y otras variables demográficas. Por su parte, Confie registra el capital institucional representado en la confianza de los ciudadanos en el Estado y en aquellas instituciones que contribuyen a resolver los problemas de la sociedad (Sudarsky, 2007).

Las diez dimensiones incluidas en el estudio fueron la participación cívica, medida por la membrecía activa con organizaciones voluntarias seculares; la confianza institucional, con referencia a instituciones del Estado como el Congreso, la Fiscalía y el propio gobierno; la solidaridad y la mutualidad, medidas por la percepción de ayuda o colaboración entre ciudadanos; las relaciones horizontales en función de cooperación y articulación entre vecinos, amigos y compañeros de trabajo; la articulación vertical, por la vinculación con organizaciones como la Iglesia, sindicatos, gremios o partidos políticos; el control social del Estado, en función del conocimiento de los mecanismos de control del Estado y su entes; la responsabilidad por lo público, medida como un concepto opuesto al clientelismo y al particularismo; la participación política, con base en las perspectivas democrática representativa y democrática participativa; e información y transparencia, con referencia a la actividad directa con medios y grado de confianza en los mismos (ver Tabla 1).

Tabla 1. Diez dimensiones y variables Barcas 20112

\begin{tabular}{|c|c|}
\hline DIMENSIONES & VARIABLES \\
\hline \multirow[b]{2}{*}{ SOLIDARIDAD Y MUTUALIDAD } & Solidaridad Manejo del Conflicto Reciprocidad \\
\hline & Atomización (-) \\
\hline \multirow{2}{*}{ RELACIONES HORIZONTALES } & Solidaridad en las relaciones horizontales \\
\hline & Actividades sociales con los del trabajo Actividades sociales con los del vecindario \\
\hline \multirow{4}{*}{$\begin{array}{c}\text { JERARQUÍA O ARTICULACIÓN } \\
\text { VERTICAL }\end{array}$} & Pertenencia a organizaciones de interés Solidaridad vertical \\
\hline & Solución vertical a problemas colectivos Hace mediación Iglesia \\
\hline & Hace mediación Gremios o Asociaciones profesionales Hace mediación Sindicatos \\
\hline & Hace mediación Partidos Políticos \\
\hline \multirow{5}{*}{ CONFIANZA INSTITUCIONAL } & Confianza en el Gobierno Confianza en los Movimientos \\
\hline & Confianza en la Iglesia \\
\hline & Confianza en la Educación Confianza en la Policía \\
\hline & Confianza en las grandes compañías \\
\hline & Honestidad y cumplimiento de la Ley Corrupción (-) \\
\hline \multirow{2}{*}{ CONTROL SOCIAL } & Confianza en las instituciones que controlan el Estado \\
\hline & Mecanismos de participación de control de la sociedad al Estado Rendición de cuentas (accountability) \\
\hline \multirow{3}{*}{ PARTICIPACIÓN CÍVICA } & Pertenencia a organizaciones Voluntarias Seculares \\
\hline & Actividades Localidad \\
\hline & Actividades Cívicas Actividades Medios \\
\hline
\end{tabular}




\begin{tabular}{|c|l|}
\hline \multirow{4}{*}{\begin{tabular}{|l|}
\multirow{4}{*}{ INFORMACIÓN Y } \\
TRANSPARENCIA
\end{tabular}} & Los colombianos no saben para dónde va el país porque nadie les informa (-) \\
\cline { 2 - 3 } & La gente tiene quien le explique los problemas públicos \\
\cline { 2 - 3 } REPUBLICANISMO CÍVICO & El Estado se esfuerza por informar a los ciudadanos \\
\cline { 2 - 3 } & $\begin{array}{l}\text { Lo medios de comunicación explican a fondo los problemas Los ciudadanos se informan para poder } \\
\text { participar }\end{array}$ \\
\cline { 2 - 3 } & Sabe en qué se van a invertir los recursos de su localidad/comuna \\
\hline \multirow{4}{*}{ MEDIOS } & Educación Política \\
\cline { 2 - 3 } & Politización \\
\cline { 2 - 3 } & Responsabilidad de lo público \\
\cline { 2 - 3 } & Particularismo (-) \\
\cline { 2 - 3 } & Clientelismo (-) \\
\hline & Confianza en los medios \\
\cline { 2 - 3 } & Actividad en los medios \\
\cline { 2 - 3 } & Los medios de comunicación explican a fondo los problemas \\
\hline
\end{tabular}

Fuente: Hurtado, García, \& Copete (2012).

Por otra parte, se incluyeron factores para analizar el contexto -análisis de causalidad no lineal o por cuadrantes del desarrollo-, para hacer una relación entre la tasa de crecimiento asociada con efectividad comunitaria y el desarrollo asociado con el logro (ver Gráfica 1). Se parte de la sociedad comunitaria que logra convertirse en sociedad cívica moderna; con estadios alternos como el "familismo amoral" caracterizado por el deterioro comunal; y la modernización antecedida por alto nivel de logro (McClelland, 1995; Putman, 1995; Woolcock, 1998).

Gráfica 1. Análisis no lineal del desarrollo

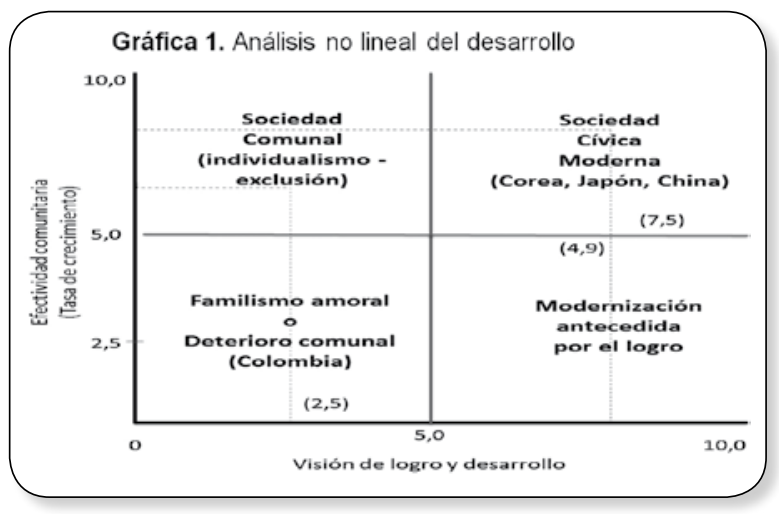

Fuente: Adaptado a partir de Sudarsky (2005).

De esta forma el análisis no lineal del desarrollo (ver Gráfica 1), en la medición del 2005, enfatiza en la interacción entre logro, civismo y modernización como factores esenciales en la transformación de una sociedad comunal donde prevalecen relaciones naturales, individualismo, exclusión y conflicto a una sociedad cívica moderna donde se combina civismo y alta participación ciudadana. La expectativa de crecimiento económico es del 2.9\% para las sociedades caracterizadas por el familismo amoral, mientras que para sociedades con visión de logro llegan a la modernización con crecimiento de $5.9 \%$ y eventualmente al $7.5 \%$, cuando se convierten en sociedades modernas y cívicas. Corea, China y Japón, son ejemplos de crecimiento económico antecedido por alto logro, como se deduce de las tasas de crecimiento de $7.5 \%$ por períodos sostenidos de 25 años. Mientras tanto Colombia presentó niveles de logro bajos que predijeron tasas de crecimiento de solo 2.5\% en 1997 (Sudarsky, 2007).

Colombia se caracteriza por el deterioro comunitario y la exclusión social, asociados con el familismo amoral (Banfield, 1958; Sudarsky, 2002; Garzón, 1998) por el persistente aislamiento y la atomización social, combinados con carencias en seguridad social, mínimo contacto con vecinos, compañeros de trabajo y representantes elegidos, como se evidencia en las mediciones con el Barcas. Esta situación invoca la sarcástica expresión de los estudios de Banfield (1958): "todo aquel que se ocupa

\footnotetext{
${ }^{2}$ Estas dimensiones han sido la guía en la aplicación de los Barcas de 1997, 2005 y 2011. Algunas variables se mantienen o reubican en las
} dimensiones. 
del bien colectivo sin una inmediata ganancia personal es considerado un fiasco". Con relación al deterioro comunal en América Latina se mencionan las organizaciones delictivas o mafias que generan beneficios propios al interior de sus organizaciones y miembros, pero con detrimento del contexto social y político, y la consecuente generación de incertidumbre, pérdidas humanas y un "capital social perverso" (Rubio, 1997).

En general, los cambios sobresalientes entre la medición de octubre de 1997 y diciembre de 2005 , registran un leve incremento del capital social a 106\%. La mayor incidencia en este resultado es el leve incremento de la solidaridad, las relaciones horizontales y las habilidades políticas. La confianza en las instituciones (Confie) cayó a un 99\%, atribuida a la caída de confianza en el congreso, la fiscalía, los gremios, la prensa y con notoria caída de confianza en la televisión. El segundo factor, fe en fuentes de información (Fenoval), subió al $133 \%$, explicado por el mayor interés ciudadano en los asuntos públicos. Se estima que este aumento es más una disposición a participar que una real utilización de los mecanismos de participación (Hurtado, 2012).

Con relación a los resultados específicos de las mediciones de 1997 y 2005, el bajo capital social colombiano está asociado con bajo nivel de confianza institucional y baja participación cívica, variables con descensos del $28 \%$ y $22 \%$ entre 1997 y 2005 . Este resultado combinado con la alta percepción de la corrupción, 73\% de los encuestados percibía el país como corrupto en 2005, origina un imperativo de realizar cambios para el mejoramiento del capital social colombiano en el corto y largo plazos (Hurtado, 2012).

Las principales fuentes de percepción de corrupción y baja confianza son el Congreso (34\%) y los partidos políticos (27\%); con porcentajes menores están gobierno, sindicatos, gremios y fiscalía. Nótese que la percepción de desconfianza y corrupción emanan de la misma cúpula encargada de dirigir y controlar al Estado colombiano; si se agrega la percepción de altos niveles de desconfianza y corrupción en los partidos, sindicatos y gremios, como entidades articuladoras entre las comunidades y las instituciones del estado, el ámbito del capital social requiere cambios estructurales para avanzar hacia una sociedad cívica moderna, considerando que la acción colectiva de los ciudadanos debe apoyarse en un conjunto de normas y organizaciones integradas (Woolcock, 2000).

Esta participación de la sociedad civil requiere acompañarse de voluntad política para mejorar la inversión social en las zonas marginadas, evitando la atomización y el desplazamiento. El gobierno de Colombia en el año 2011, había registrado más de 3.7 millones de desplazados en el país como consecuencia del conflicto armado, ocupando el primer lugar en número de personas desplazadas, según estudios de la ONU (Acnur, 2013).

\section{LA CONVERGENCIA DE LA DESCONFIANZA Y LA CORRUPCIÓN EN EL BAJO CAPITAL SOCIAL}

Los resultados y análisis hechos con base en la aplicación del BARCAS en 1997 y 2005 fueron predictivos de la situación actual de desconfianza y corrupción. Así, en el informe de competitividad mundial 2011 - 2012, Colombia está clasificada en el puesto 118 entre 142 países con mayor corrupción; y en el puesto 89 sobre 142 países con mayor despilfarro de recursos públicos (Sala-i-Martin \& Schwab, 2011). Igualmente en el informe de Transparencia Internacional, ONG con sede en Alemania, Colombia ocupó el puesto 94 entre 176 países del mundo, en el año 2012, con una calificación de 36/100.

La corrupción aparece como el factor más problemático para hacer negocios en Colombia, seguido de la deficiente infraestructura, el acceso al financiamiento, las regulaciones fiscales y la deficiente infraestructura, de acuerdo con El Foro Económico Mundial y el "El Reporte de Competitividad Global 2010 - 2011" (Sala-i-Martin \& Schwab, 2011). Por su parte,
Junio - Diciembre 2013 ISSN 0122-820X PP: 43-60 
el ex - ministro y columnista Rudolf Hommes haciendo referencia a las alcaldías exitosas en Colombia, afirma: ".... son lamentablemente las excepciones en un universo plagado de malas administraciones locales en manos de incapaces, de clientelistas, de irresponsables, guerrilleros o mafiosos" (Hommes, 2013).

El actual Auditor General de la Nación en su discurso de posesión y en la cartilla institucional de prevención y control social expresa: "..., la viabilidad de nuestro país y sus instituciones, no se sustenta en las pródigas riquezas naturales, ni en el cabal ejercicio constitucional y ordenamiento jurídico del estado social de derecho, sino en combatir la corrupción" (Melo, 2013). Está tan arraigada la corrupción que existen nociones justificativas y eufemísticas como "aprobación privada de los bienes públicos" o como "una simple manifestación de mercantilismo individualista en el marco del liberalismo capitalista". Argumentos muy extendidos en el ámbito latinoamericano (Neiva, 1993).

Por su parte, el informe presentado en la Universidad de los Andes por la agencia local del "Barómetro para las Américas" el 20 de febrero de 2013, registra que Colombia es el país donde menos se confía en los partidos políticos y no se tiene notoria simpatía por alguno en particular. La falta de confianza alcanza el $69 \%$ y solo $31 \%$ de los encuestados tiene simpatía por algún partido político. Contrastan estos datos con el 63.4\% en Estados Unidos y el $61.1 \%$ de confianza en República Dominicana (Rodríguez Raga \& Seligson, 2013). Esta desconfianza se hace evidente ante las denuncias contra apreciable número de congresistas condenados o sometidos a procesos judiciales, más del 30\% por delinquir en contra de la estabilidad democrática y el patrimonio público del país (Nizkor, 2011).

Mediante la asociación de estos estudios con las mediciones de capital social hechas en Colombia (1997, 2005 y 2011), se establece que una de las causas asociadas con el bajo capital social es el alto grado de desconfianza y corrupción en las instituciones del Estado, con el agravante que algún sector de la opinión pública colombiana (21.1\%) parece justificar los sobornos por la proliferación de normas anticorrupción que son cotidianamente incumplidas (Ryan, 2013).

\section{TERCERA MEDICIÓN DEL CAPITAL SOCIAL EN COLOMBIA - BARCAS 2011}

La tercera medición con el BARCAS se realiza por un equipo presidido por David Hurtado en el año 2011 y patrocinada por la Fundación Restrepo Barco, la misma entidad financiadora del Barcas 2005. En primer lugar, esta medición reordenó las dimensiones que conforman los factores Ksocial, Confie y Fenoval, con relación al Barcas de 2005. El primer grupo se organiza al considerar que las interacciones sociales y el tejido social son la esencia del capital social (Hurtado et al., 2012), lo que origina la reagrupación de las dimensiones solidaridad, relaciones horizontales y jerarquía en el factor Ksocial.

Por su parte, el segundo grupo se integra con las dimensiones que relacionan la sociedad con sus instituciones, de esta manera el factor Confie se conforma con las dimensiones confianza institucional, control social, participación cívica, y participación política. El tercer grupo se integra por las dimensiones: información y transparencia, republicanismo cívico y medios, agrupados en Fenoval. Los resultados generales de la tercera medición Barcas 2011, presentan una disminución de los tres factores: Ksocial, Confie y Fenoval. Mientras que en la segunda medición realizada por el profesor Sudarsky en 2005, estos factores habían aumentado levemente, con la excepción de Confie, factor que ha disminuido desde la primera medición en 1997.

Los cambios más notorios están en la percepción de corrupción con un incremento del 8\% al pasar de 6.8/10 en 2005 a 7.4/10 en 2011, manteniéndose en un nivel muy alto desde la primera medición de 1997. Igualmente, la solidaridad tuvo una caída del 74\% entre 2005 y 2011, por el aumento de las variables atomización social, disminución de ayuda del Estado 
y pérdida de representación o eslabonamiento legislativo. El incremento de la informalidad laboral contribuyó con la caída de la solidaridad al registrarse que el $52 \%$ de personas que cotizaban en fondos de pensiones bajó a un 24\% entre 2005 y 2011 (Hurtado, 2012).

La variable pérdida de confianza interpersonal, se mantiene en un nivel muy bajo, $10 \%$ (1997), 14\% (2005) y 11\% (2011), como consecuencia de una disminución de "las soluciones a problemas entre familiares y vecinos"; y el aumento de las personas que expresaron "preferir buscar un agente externo para dar la solución a los problemas colectivos" (Hurtado, 2012).

En síntesis, se registra disminución del Ksocial entre 2005 y 2011 por las disminuciones en solidaridad $26 \%$, relaciones horizontales $20 \%$, jerarquía 16\% y el aumento de la atomización ciudadana $27 \%$. Esto significa que la acción colectiva y democrática, así como el buen desempeño institucional tuvieron notoria desmejoría en el período comprendido entre las mediciones de 2005 y 2011.

Con relación a la confianza institucional (Confie) tuvo una disminución superior al 6\% en la última medición con relación al 2005. Las causas fueron pérdida de confianza en Instituciones educativas (18\%), grandes compañías $(12 \%)$, aspectos legales $(10 \%)$ y en el gobierno (11\%). Además, del incremento de la percepción de corrupción en $8 \%$ con relación al 2005.

Llaman la atención las variables participación política y control social del Estado por su disminución en $12 \%$ en 2011, frente al aumento del 29\% registrado entre 1997 y 2005 . Las causas son la disminución del uso de mecanismos de participación ciudadana en salud, desarrollo rural y veedurías ciudadanas (Hurtado, 2012).

Por su parte, fe en los medios Fenoval registró una estruendosa caída del 340\% con relación al 2005, cuando se registró un aumento del $77 \%$ entre 1997 y 2005 . Este impactante retroceso se debe a caídas de las variables in- formación y transparencia (63\%) y republicanismo cívico (42\%); y en los items: "la gente no tiene quien le explique los problemas" o carencia de explicación de los problemas por parte de los medios y el propio Estado (Hurtado, 2012).

Los ítems con incidencia negativa en Ksocial fueron las ayudas del Estado, las organizaciones humanitarias y ayuda de los vecinos. Por su parte, los ítems que afectaron Confie fueron el uso de mecanismos de participación, confian$z a$ en instituciones de control y pertenencia a organizaciones seculares. En la estruendosa caída de Fenoval, las mayores causas fueron: los medios explican los problemas a profundidad, el estado informa, la gente tiene quien le explique los problemas públicos y los ciudadanos se informan para participar. Estos ítems de Fenoval son afectados por la baja confianza en el gobierno y "los colombianos no saben para dónde va el país".

Con relación a las variables exógenas, los grupos etarios marcaron diferencias, los jóvenes registraron menor participación en los asuntos públicos, al igual que las mujeres, lo que confirma la relegación femenina en la vida pública (Hurtado, 2012).

La educación tiene alta incidencia en la participación ciudadana, lo cual valida la denominada "movilización cognitiva", mencionada por Sudarsky en la medición de 2005 y que se genera a partir del acceso a la educación media. Por su parte, el tamaño del municipio presenta alta influencia, registrándose mayores niveles de capital social en comunidades pequeñas y por presunta mayor eficiencia de las unidades administrativas en municipios menores a quinientos mil habitantes (Hurtado, 2012).

Con relación a las regiones y unidades administrativas, Bucaramanga presentó el capital social más alto (66\%), con relación al promedio del país, en contraste con la región del Tolima, Pacífico, Orinoquía y Eje Cafetero con los niveles más bajos. A Bucaramanga se suman Santander, Barranquilla y Cartagena, con niveles altos. Norte de Santander
Junio - Diciembre 2013 ISSN 0122-820X

PP: $43-60$

\section{1}


No. 2

Junio - Diciembre 2013 ISSN 0122-820X

PP: $43-60$

que había tenido buen resultado en el período 1997 - 2005, presentó la caída más dramática de Ksocial en el período 2005 - 2011. En este departamento se encuentra la región del Catatumbo integrada por ocho municipios y doscientos siete mil habitantes - según estadísticas del Dane 2006 -, un ejemplo típico de desplazamiento y marginamiento socioeconómico, no obstante su biodiversidad, riqueza petrolera y agrícola. Sobre esta región se abre una esperanza de gran impulso gubernamental pues según el boletín de la Dirección $\mathrm{Na}$ cional de Planeación, enero 16 de 2013, se aprobaron $\$ 1.7$ billones para el desarrollo vial e integral (Colombia, DNP , 2013).

Al tomar en cuenta estas mediciones y sus resultados es conveniente articular las relaciones entre la sociedad y el Estado con ámbitos de confianza que generen gobernabilidad, orden social y prosperidad económica como factores interdependientes para el mejoramiento del capital social (Woolcock \& Narayan, 2000). Además, el capital social local está atomizado en diferentes grupos políticos, sociales y económicos, con alta incidencia en el marginamiento y la exclusión socioeconómica, lo cual influye en el mantenimiento de conflictos actuales y latentes (Woolcock, 2000). Se viven momentos propicios para que los grupos excluidos y de activistas sociales promuevan grandes coaliciones en torno a los partidarios de acrecentar el capital social, con acceso a los centros de poder (Fox \& Brown, 1998) y por ende lograr recursos y derechos hasta ahora negados o de los cuales han sido despojados.
Asimismo, existen informes que ubican a Colombia entre los países más inequitativos del mundo por factores como desigualdad social, marginamiento político y económico, altos índices de pobreza e informalidad laboral - esta última registraba el $51.3 \%$ a diciembre de 2011. No obstante, este preocupante panorama político, social y económico se mantiene el respaldo a los partidos y al sistema democrático, lo cual puede ser explicado por el alto clientelismo registrado en las mediciones de capital social (Portafolio, co, 2013).

\section{APORTES Y AVANCES DE LAS MEDICIONES DEL CAPITAL SOCIAL 1997 - 2012}

\subsection{Evaluación de la estructura del capital social en Colombia}

A continuación se relaciona el Informe del Barómetro de las Américas 2012 con el Barcas 2005, este último en la dimensión eslabonamiento legislativo - acercamiento de la gente con sus líderes políticos-, establece que el 29\% de los electores saben quién es su representante y solamente 3\% identifica a su "representante de confianza" (Rodríguez Raga \& Seligson, 2013; Sudarsky, 2007). En concordancia el Informe del Barómetro para las Américas 2012, registra que la falta de confianza en los partidos alcanza el $69 \%$ y solo $31 \%$ de los encuestados tiene simpatía por algún partido político.

Con base en estos datos, se puede afirmar que Colombia tiene -lo que podríamos denominar- un "estructuralismo entrópico" configurado, por una parte, por las comunidades del sector rural y las poblaciones urbanas marginadas con bajas relaciones verticales y excluidas de otros sistemas económicos y sociales; y por otra parte, la élite urbana configurada por el clientelismo y el particularismo urbano. Esta denominación se inspira en la concepción estructuralista que distingue los elementos organizativos por su posición con relación a una totalidad - estructuralismo- y al mismo tiempo produce pérdida de energía por aislamiento - entropía- de los elementos como producto de esa diferenciación (Chiavenatto, 1998), fomentando la atomización urbano - rural y el distanciamiento entre la dirigencia política y la sociedad civil.

Con respecto a la articulación vertical o pertenencia a organizaciones, llaman la atención los sindicatos, gremios, iglesia y en general las organizaciones cívicas seculares por su pérdida continúa de afiliados o seguidores. Se registra que un $43 \%$ de la población secular mayor de 18 años no pertenece a organización alguna, la mayor parte pertenecientes a estratos bajos 
del sector urbano (Sudarsky, 2007).

Con relación a los sindicatos, entre las causas de pérdida de membrecía están la inseguridad política, las contradicciones sindicales internas (matizadas por intereses partidistas y económicos), el clientelismo y el corporativismo social; este último se califica así por la firma de acuerdos entre dignatarios sindicales y líderes empresariales a espaldas de la base de trabajadores.

Las organizaciones sindicales son el eslabón para atenuar la alta informalidad laboral, el bajo nivel de ingresos de la población, la inequidad social y la pobreza, cuyos datos para Colombia registran veinte millones de personas que viven en la pobreza y siete millones que habitan en la indigencia o la pobreza extrema, en el año 2009 (Orozco, 2010). Esta posibilidad de eslabonamiento sindical deberá darse a partir del abandono de la práctica del corporativismo excluyente - asociación de sindicatos y gremios - y el clientelismo relacionado con la corrupción, como orientación básica de la política del país. Es pertinente acotar que la disminución del clientelismo se logra mejorando la educación a partir del nivel medio y la formación política, ámbitos exógenos del capital social (Sudarsky, 2007).

Con relación a la Iglesia, ha disminuido la membrecía pero ha avanzado en pluralidad de grupos o sectas religiosas. En el marco de la nueva Constitución, en el artículo 19, se consagra la libertad de culto y se desconoce la supremacía de la iglesia católica (Kalipedia, 2013), al rezar que "se garantiza la libertad de cultos" y que "todas las organizaciones religiosas e iglesias son igualmente libres ante la ley" (Colombia, Constitución 1991a ). No obstante, las organizaciones religiosas continúan registrando el mayor número de miembros por encima de las organizaciones seculares que han tenido poco crecimiento relativo. Los clubes recreativos, deportivos y culturales tienen una membrecía del $22 \%$, superior al $21 \%$ de las organizaciones educativas, $16 \%$ de las juntas de acción comunal y $15 \%$ de los grupos políticos (Sudarsky, 2007). En el escenario mundial el tema religioso cobra gran importancia por su influencia en la cultura y el desarrollo. Así en algunas sociedades como Nigeria, el 95\% de los habitantes considera a "dios muy importante en sus vidas" mientras que para Japón y Alemania solo el 5\% lo considera así, lo cual es un inmenso contraste (Inglehart, 2002).

Por otra parte, los grupos indígenas, grupos marginados y en general los países de América Latina se caracterizan por altos niveles de solidaridad y proclividad a la asociación pero los altos niveles de pobreza, falta de recursos y acceso a los centros de poder, inhiben alcanzar mejores resultados como los obtenidos en otros países que sí tienen apoyo del Estado y organizaciones sociales. Es necesario cambiar las reglas de juego a favor de estas comunidades marginadas (Narayan-Parker, 1999), y aceptar, como lo expresa Kliksberg (2006, p. 30), que "el capital social y la cultura han comenzado a instalarse como el centro del debate sobre el desarrollo...", para superar el reduccionismo económico e incluir las dimensiones políticas, institucionales y culturales, haciendo eco al clamor de un reexamen profundo como lo solicita el premio nobel de economía 1998 - Amartya Sen -, al mismo tiempo que cuestiona el modelo actual de desarrollo de "sangre, sudor y lágrimas" (Kliksberg, 1999).

En esta misma perspectiva, las mediciones del Barcas (1997, 2005 y 2011), establecen que las poblaciones de menor tamaño tienen mayor disposición a generar capital social, sin embargo estas regiones no son las que tienen mayor desarrollo socio económico. En forma específica, las regiones de Cauca- Huila, Cundinamarca y Cesar-Guajira- Magdalena presentaron los niveles más altos de capital social en la medición del 2011 con relación al promedio nacional, pero menor nivel de desarrollo. En forma similar, la región del Pacífico y parte de la Costa Atlántica registran niveles superiores de participación ciudadana, factor determinante del capital social, pero igualmente su nivel de desarrollo socio económico está por debajo de las regiones del área andina (Eje Cafetero, Bogotá y Boyacá), cuyo capital social presenta niveles inferiores en un $40 \%$
Junio - Diciembre 2013

ISSN 0122-820X

PP: 43-60 
Vol. 18

No. 2

Junio - Diciembre 2013 ISSN 0122-820X

PP: 43-60 al promedio nacional (Hurtado et al., 2012), pero con mayor nivel de desarrollo económico por la mayor inversión social recibida por parte del Estado.

\subsection{Desarrollo del capital social en función del nivel de necesidades}

De acuerdo con el artículo "Capital Social: Mediciones y Consecuencias" (Putnam, 2001), el autor inspirado en su propio libro "Colapso y Resurgimiento de la Comunidad Americana" (1995), hace una correlación entre el nivel de ingresos de la población y las diferentes expresiones caritativas y filantrópicas de los norteamericanos, cuyas contribuciones estuvieron acordes con el nivel de ingresos de la población en el siglo XX. Se trata de analizar el capital social con base en el contexto socioeconómico y las necesidades individuales.

Se describe como durante la Gran Depresión (1929 - 1940) o profunda recesión económica mundial (Krugman, 1999) las contribuciones filantrópicas disminuyeron, mientras que en el período de la postguerra aumentaron. Esta pragmática correlación entre capital social y nivel de ingresos, permite hacer un análisis similar con el nivel de necesidades satisfechas de la población, teniendo como referencia la teoría de la "jerarquía de necesidades" (Maslow, 1954); y las relaciones entre los ciudadanos y las instituciones descritas en el capital social estructural (Woolcock, 1998) (ver Figura 1).
Figura 1. Desarrollo del capital social en función de la jerarquía de necesidades y el capital social estructural.

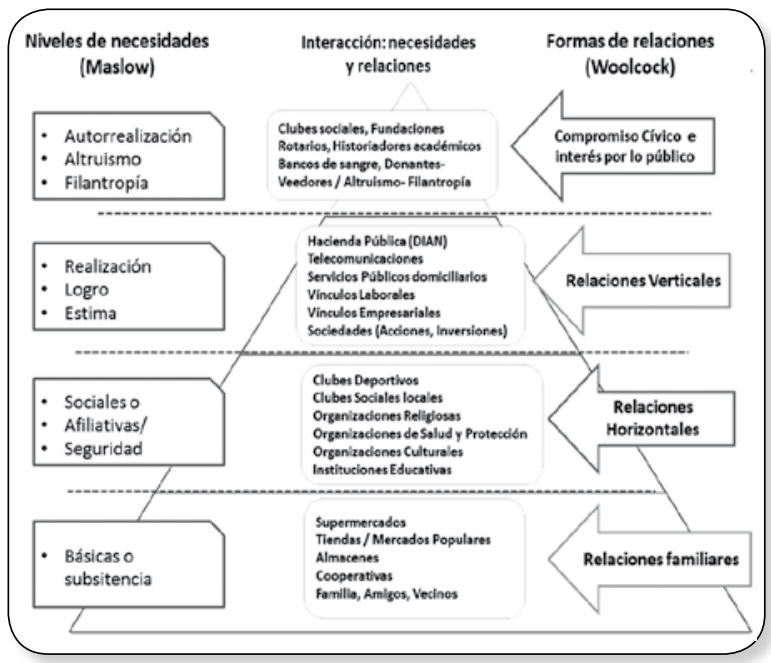

Fuente: Elaboración a partir de Maslow (1954) y Woolcock (1998)

La figura 1 describe cómo a medida que los ciudadanos superan sus necesidades básicas, sociales y egocéntricas, convertidas en motivadores, amplían y diversifican sus vínculos personales, sociales y estructurales, acumulando capital social en cada nivel de necesidad que se convierte en motivador (Maslow, 1954). Solamente en el nivel superior, egocentrismo o auto realización, emerge en los ciudadanos un mayor compromiso con el civismo y con lo público, generando entidades culturales, cívicas y sociales de cooperación espontánea y generosa como bancos de sangre, organizaciones rotarias, fundaciones, entre otras.

En el nivel de subsistencia, columna central de la Figura 1, la gente limita su participación a actividades muy cercanas con el tendero que le fía o con el familiar o amigo que lo ampara o subsidia. Esta reflexión lleva a plantear que el desarrollo del capital social puede caer en el llamado "circulo vicioso del sub desarrollo" (Martínez, 2001), cuya superación requiere mejorar las condiciones sociales, políticas y económicas; así como un despegue o gran impulso ("big push") apoyado en grandes inversiones de capital como instrumento principal del desarrollo (González, 2006) con base en una alianza entre el Estado y la sociedad civil -or- 
ganización en función de valores de autonomía y democracia, independiente del Estado que articula fuerzas sociales que se contraponen a la sociedad mercantilista no controlada (Cohen, 2001).

Este planteamiento es coherente con los resultados de las mediciones hechas por el Barcas en Colombia, donde existen regiones geográficas con alta proclividad a confiar y participar, pero presentan bajo desarrollo económico y la consecuente necesidad de recibir un gran impulso en infraestructura social para superar el marginamiento; al igual que potencializar su proclividad a la solidaridad y la cooperación, lo cual ha sido posible en otros escenarios con la sinergia entre comunidades, redes sociales, instituciones y la acción conjunta entre ellas (Woolcock, 2000).

En este análisis cobra importancia la correlación entre la jerarquía de las necesidades y las dimensiones del capital social, por cuanto se encuentran ligados en lo económico y lo social. Significa que el capital social encuentra un común denominador con el desarrollo económico en la búsqueda del desarrollo social, lo cual es coherente con la influencia del desarrollo del individuo en el desarrollo de las organizaciones (Coleman, 1988).

\subsection{Pertinencia de las dimensiones aplica- das en la medición del capital social en Co- lombia}

Para hacer un análisis comparativo entre las mediciones de capital social en Colombia con otros países, se diseña la Tabla 1 apoyada en el texto - resumen: "Capital Social: Conceptualización, Enfoques y Mediciones" (Portales \& García, 2009), específicamente en su anexo No. 1, donde se analizan las dimensiones de medición aplicadas en diez territorios geográficos a nivel mundial. En esta figura se registra el Barcas de Colombia en la primera columna como referente principal.

Tabla 2. Dimensiones utilizadas en las mediciones de ksocial a nivel mundial (1997-2008)

\begin{tabular}{|c|c|c|c|c|c|c|c|c|c|c|c|c|}
\hline & 1 & 2 & 3 & 4 & 5 & 6 & 7 & 8 & 9 & 10 & & \\
\hline $\begin{array}{l}\text { INSTRUMENTOS= } \\
\text { PAIS/REGIÓN } \rightarrow\end{array}$ & 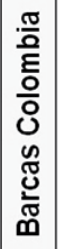 & 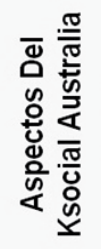 & 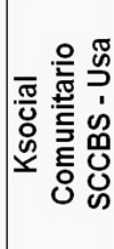 & 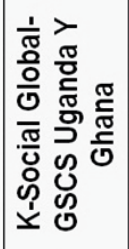 & 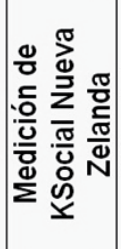 & 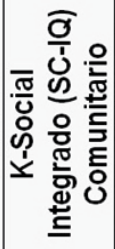 & 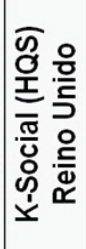 & 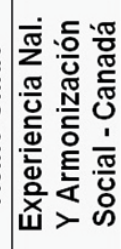 & 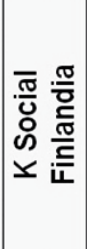 & 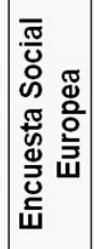 & 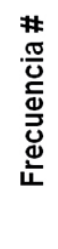 & 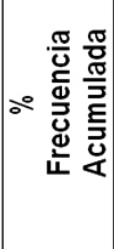 \\
\hline \multicolumn{13}{|l|}{$\begin{array}{l}\text { DIMESIONES } \\
\text { UTILIZADAS } \downarrow\end{array}$} \\
\hline $\begin{array}{l}\text { 1. Participación } \\
\text { Civica }\end{array}$ & $x$ & $\mathrm{x}$ & $x$ & $\mathrm{x}$ & & & $x$ & $\mathrm{x}$ & $\mathrm{x}$ & $x$ & 8 & 12.3 \\
\hline $\begin{array}{l}\text { 2. Confianza } \\
\text { Institucional }\end{array}$ & $x$ & $x$ & $\mathrm{x}$ & $x$ & $\mathrm{x}$ & $\mathrm{x}$ & $\mathrm{x}$ & $x$ & $\mathrm{x}$ & $x$ & 18 & 27.6 \\
\hline $\begin{array}{l}\text { 3. Solidaridad y } \\
\text { Mutualidad }\end{array}$ & $x$ & $x$ & $x$ & $x$ & & $\mathrm{x}$ & $x$ & $\mathrm{x}$ & $x$ & $x$ & 27 & 41.5. \\
\hline $\begin{array}{l}\text { 4. Relaciones } \\
\text { Horizontales }\end{array}$ & $\mathrm{x}$ & $x$ & $\mathrm{x}$ & $x$ & $x$ & $\mathrm{x}$ & $x$ & $x$ & $\mathrm{x}$ & $\mathrm{x}$ & 37 & 56.9 \\
\hline $\begin{array}{l}\text { 5. Articulación } \\
\text { vertical }\end{array}$ & $x$ & $x$ & $x$ & $x$ & $x$ & & $x$ & & $x$ & & 44 & 67.6 \\
\hline 6. Control Social & $x$ & - & - & & & & $x$ & & $\mathrm{x}$ & $x$ & 48 & 73.8 \\
\hline $\begin{array}{l}\text { 7. Republicanismo } \\
\text { Cívico }\end{array}$ & $x$ & & $x$ & & & $x$ & & & $x$ & $x$ & 53 & 81.5 \\
\hline $\begin{array}{l}\text { 8. Participación } \\
\text { Política }\end{array}$ & $\mathrm{x}$ & $\mathrm{x}$ & $x$ & & $x$ & & & & $\mathrm{x}$ & & 58 & 89.2 \\
\hline $\begin{array}{l}\text { 9. Inform. y } \\
\text { Transparencia }\end{array}$ & $x$ & & $x$ & $x$ & & $\mathrm{x}$ & & & & $\mathrm{x}$ & 63 & 96.9 \\
\hline 10. Medios & $\mathrm{x}$ & - & - & & & & & & $x$ & & 65 & 100.0 \\
\hline
\end{tabular}

Fuente: Elaboración a partir de "Capital Social: Conceptualización, Enfoques y Mediciones" (Portales \& García, 2009) y el Barcas (2011). 
Vol. 18

No. 2

Junio - Diciembre 2013 ISSN 0122-820X

PP: 43-60
El propósito inicial de la Tabla 2, es asimilar las dimensiones utilizadas por el Barcas de Colombia a las dimensiones más utilizadas para medir capital social a nivel universal. A partir de esta asimilación se analiza la frecuencia del uso de las dimensiones y la justificación de las diferentes denominaciones de acuerdo con la diversidad cultural, política y educativa de cada país.

Con relación a la frecuencia se establece que el $67.6 \%$ de las mediciones utilizaron las dimensiones: participación cívica, confianza institucional, solidaridad y mutualidad, relaciones horizontales y relaciones verticales o jerarquía. Es decir, el gran soporte de la conceptualización y aplicación del capital social está en las relaciones personales y estructurales del capital social, encontrándose que las dimensiones confianza, solidaridad y mutualidad están incluidas en el 100\% de las diez mediciones internacionales registradas en la tabla 2, realizadas entre 1997 y 2008. Se deduce el uso fundamental de estas dimensiones en cualquier medición y amplía la validez y la universalidad del concepto de capital social como nuevo paradigma de investigación.

A su vez, cada región tiene dimensiones particulares que obedecen a condiciones locales; es oportuno invocar los postulados del pensamiento complejo (Morin, 1997), porque se trata de una diversidad incluyente de la complejidad o principio holístico del pensamiento integrador. Por ejemplo, es comprensible que la "encuesta comunitaria" hecha para medir capital social en los Estados Unidos en el año 2000 , incluya la dimensión "confianza interracial", por las razones históricas locales de su confrontación étnica. $\mathrm{O}$ en esta misma medición se incluya la dimensión "compromiso basado en la fe", recogiendo la característica particular de los grupos religiosos politeístas, en su mayoría comprometidos con el desarrolo pragmático comunitario. Allí la secularización para incrementar el capital social no es una preocupación, como sí lo puede ser en nuestro país donde la secularización y la libertad religiosa crecen progresivamente a partir de la globalización y la Constitución de 1991 (Bidegain, 2005).
Por su parte, las mediciones hechas en Tanzania (2004) y Reino Unido (2003) incluyen en forma explícita la dimensión "inclusión social"; aunque se trata de dos países con niveles distintos de desarrollo económico, tienen formas distintas de atomización social, porque para Tanzania esta dimensión apunta al nivel de ingresos de la población y en el Reino Unido la misma dimensión apunta a los aspectos étnico y social. Por su parte, al incluir esta dimensión para medir el capital social en nuestro país, el resultado caería dramáticamente porque "Colombia es una sociedad con profundas raíces de exclusión social" (Garay, 2008, p.1); donde existe una alta deuda con la inclusión social, caracterizada por un sistema integral de justicia, protección, saneamiento financiero y seguridad social.

Hace contraste la baja frecuencia de las dimensiones "control social" y "medios" del Barcas con los otros países y regiones donde estas dimensiones están implícitas en denominaciones como empoderamiento, información y comunicación, actitudes y valores, normas generalizadas y redes sociales. Una aproximación es que en los escenarios internacionales referenciados existe menos preocupación por el control social y los medios de información porque estos se soportan en culturas o valores con mayor proclividad a la transparencia y la ética de lo público, entendiendo la ética pública como una disciplina con principios y valores deseables en las personas que desempeñan una función pública (Bautista, 2009). Mientras tanto para Colombia los resultados de las mediciones sobre corrupción hechas por organizaciones internacionales (Transparencia Internacional/Barómetro para las Américas), predicen alta incidencia en el bajo capital social porque "la corrupción mina la confianza de la gente es sus instituciones" (Caiden, 2001).

\section{CONCLUSIONES}

Colombia presenta grandes avances en el desarrollo del concepto capital social y su aplicación como nuevo tema de investigación 
universal en los campos de la sociología y la economía. Se analizan tres trabajos de investigación desarrollados en Colombia con el instrumento Barcas (1997, 2005 y 2011), considerando factores, dimensiones, variables e ítems en sendas muestras representativas de más de tres mil participantes. Se registra una acumulación de conocimientos y resultados coherentes para mejorar la interacción entre la sociedad civil y las instituciones del Estado, en función de mejorar el bajo capital social registrado en tres mediciones hechas en nuestro país durante más de quince años de investigaciones.

Entre las causas del bajo capital social en Colombia está la deficiente tarea cumplida por las organizaciones articuladoras o llamadas a hacer "puente" entre las comunidades y las instituciones del Estado. La disminución de membrecía en sindicatos, partidos políticos, procesos electorales y organizaciones seculares, así como el aislamiento o atomización ciudadana, como lo muestran los resultados de las mediciones, son indicios de la cuestionada función que cumplen estas organizaciones.

Además, estos resultados muestran que la credibilidad ciudadana es afectada por la poca confianza en las instituciones del Estado, incluyendo al propio gobierno, al Congreso, la Fiscalía y los organismos de control. También, se registra la carencia de información oportuna, suficiente y confiable para participar o responsabilizarse de asuntos públicos. Otras causas del bajo capital social son las pocas expectativas de la gente en conseguir ayuda de la sociedad a través de la solidaridad, la cooperación y la amistad con vecinos y compañeros de trabajo. Las relaciones entre la sociedad y la institucionalidad requieren reconstruirse para detener su progresivo deterioro.

Por su parte, como una luz de esperanza, se erige la Constitución de 1991 para elevar la participación ciudadana a través del control social del Estado, con instrumentos como las veedurías, la rendición de cuentas, los derechos de petición, entre otros. Las cortes judiciales, la prensa y las entidades educativas son instituciones que mantienen altas expectativas para el mejoramiento del capital social.

En esta recuperación del tejido social las organizaciones nacionales e internacionales humanitarias tienen amplio trabajo comunitario, tal como la cohesión de familiares, vecinos, compañeros de labores y la búsqueda de soluciones locales a los problemas colectivos. Corresponde al gobierno contrarrestar los efectos negativos de las malas prácticas de los servidores públicos como el oportunismo, el clientelismo y la alta corrupción, así como mantener y ampliar los espacios de participación de la sociedad civil.

En este momento histórico, cuando Colombia se asemeja más a una sociedad comunal, alejada del modelo de sociedad moderna, matizada por la participación y ética frente a lo públi$\mathrm{co}$, se requiere una sinergia entre la sociedad civil, los gremios, los partidos políticos y las instituciones del Estado para el acercamiento de las regiones marginadas con los centros de decisión del país.

Estos cambios estructurales y sociales son parte del desarrollo del capital social en el país con el propósito de mejorar el desarrollo económico sostenible, pacificar las luchas políticas, reformar la justicia, la educación, la salud, el régimen de pensiones y en general encontrar procesos de inclusión para los colombianos marginados del sistema productivo, al igual que contrarrestar el desempleo, el desplazamiento, la inequidad social, la desnutrición, la violencia, la informalidad laboral y el cuestionamiento de la comunidad internacional.

\section{AGRADECIMIENTOS}

Al grupo organizador del Diplomado de Investigación (2012 - 2013) de la Universidad Francisco de Paula Santander, encabezado por los profesores Stella Arenas y Gabriel Peña. Especial reconocimiento a Jairo Alexander Hernández, licenciado en historia de la Universidad del Valle y asesor metodológico del trabajo.
Junio - Diciembre 2013 ISSN 0122-820X PP: 43-60 
No. 2

Junio - Diciembre 2013

ISSN 0122-820X

PP: 43-60

Acnur (2013). Desplazamiento interno en Colombia. Consultado 20 de abril de 2013, desde, http://www.acnur.org/t3/operaciones/ situacion-colombia/desplazamiento-internoen-colombia/

Bautista, Oscar Diego (2009). Etica para corruptos. "Una forma de prevenir la corrupción en los gobiernos y administradores públicos" (pp. 164. Madrid: Desclée De Bower.

Banfield, Edward. (1958). The moral basis of a backward society. Glencoe, Illinois: The Free Press.

Bourdieu, P. (1985). "The forms of capital". En Handbook of Theory and Research for the Sociology Education (pp. 241-258). Nueva York: Greenwood.

Bidegaín, A. M. (2005). Introducción. En Globalización y diversidad religiosa en Colombia (pp. 13-30). Bogotá: Universidad Nacional de Colombia, Facultad de Ciencias Humanas.

Caiden, Gerald. (2001). Tendencias actuales en la ética del servicio público. Gestión y Política Pública, 2(10), 233-252

Chiavenato, Idalberto. (1998). Introducción a la teoría general de la administración (4a ed.). Bogotá: McGraw-Hill.

Cohen, Jean \& Copete, Andrés. (2001). Sociedad civil y teoría política. Fondo de Cultura Económica, 556 - 635. México. Consultado, abril 20, 2013. http://insumisos.com/lecturasinsumisas/SOCIEDAD\%20CIVIL\%20Y\%20 TEORIA\%2OPOLITICA.pdf

Coleman, James Samuel. (1988). "Social capital in the creation of human capital". En American journal of sociology, 94, 95-120.

Coleman, James Samuel. (1990). Foundations of social theory. Cambridge: Harvard University Press.

Colombia (1991a). Constitución Política de Colombia. Secretaría del Senado [recurso online]. Consultado abril 2013 desde http:// www.secretariasenado.gov.co/senado/basedoc/cp/constitucion_politica_1991.html

(1991b). Constitución Política de Colombia. Prólogo reflexiones sobre nuestra constitución por Carlos Lemos Simmonds. Bogotá: Editorial Panamericana.

Colombia. Dirección Nacional de Planeación. (2013). Comunicado de prensa. Conpes aprueba 1.7 billones para el Catatumbo. Consultado marzo 27 de 2013 desde https://www. dnp.gov.co/LinkClick.aspx? fileticket $=\mathrm{Ni} 5 \mathrm{bY}$ pJZ1T8\%3d\&tabid $=1658$

Fox, Jonathan A, \& Brown, L David. (1998). The struggle for accountability: the World Bank, NGOs, and grassroots movements. Cambridge: MIT press.

Garay, L. J. (2008). En torno a la economía política de la exclusión social en Colombia. Revista de Economía Institucional, 5(8), 15-31.

Garzón, Adela. (1998). "Familismo y Creencias Políticas". En Psicología Política, 101 - 128.

González, Mario. (2006). Una gráfica de la teoría del desarrollo. La Habana: mgarencibia. Consultado, marzo 29 de 2013. http:// www.eumed.net/libros-gratis/2006a/mgades/le.htm

Hanifan, Lyda J. (1916). "The Rural School Community Center". Annals of the American Academy of Political and Social Science, 67, 130-138.

Hurtado, David, García, Diana, \& Copete, Andrés. (2012). Tercera medición de Capital Social en Colombia BARCAS 2011 (pp. 1-41). Bogotá: Fundación Antonio Restrepo.

Hommes, Rudolf. (2013). Supervisión Municipal, Consultado marzo de 2013, desde http://www.laopinion.com.co/demo/index. php?option $=$ com_content\&task $=$ view\&id $=$ $416548 \&$ Itemid $=86$

Inglehart, Ronald. (2002). "Modernidad y Cambio Cultural". En Reflexiones sobre la Investigación en Ciencias Sociales y Políticas, Memorias Seminario Octubre, 2002 (pp. 171 
- 197). Bogotá: Universidad Nacional.

Kalipedia (2013). Libertad de cultos en la Constitución Política de Colombia. Consultado 10 de septiembre de 2013 desde, http://co.kalipedia.com/historia-colombia/ tema/democracia-convivencia-derechoshumanos/libertad-cultos-constitucion-politica.html? x1 = 20080803klphishco_11. $\mathrm{Kes} \& \mathrm{x}=20080803 \mathrm{klphish}$ co_12.Kes

Kliksberg, B. (2010). "Más ética, más desarrollo" (6th ed.). Buenos Aires: INAP.

Kliksberg, B. (1999). Capital social y cultura claves esenciales. Revista de la CEPAL, 69, 85.

Krugman, P. (1999). De vuelta a la economía de la gran depresión. Bogotá: Norma.

Lucas Jr, Robert E. (1988). "On the mechanics of economic development". Journal of Monetary Economics, 22 (1), 3-42.

MacClelland, David. (1995). Power: The inner experience. Oxford, England: Irvington.

Martínez, Juan. (2001). "El crecimiento económico en la economía de mercado, virtudes e inconvenientes". Consultado del 29 de marzo de 2012. http://www.eumed.net/cursecon/18/ el_circulo_vicioso_del_subdesarr.htm

Maslow, Abraham H. (1954). Motivation and personality (1st ed.). New York,: Harper.

Meier, Gerald M. (1984). Leading issues in economic development (4a ed.). New York: Oxford University Press.

Melo, Luis Arturo. (2013). "iReyes de burlas?", Consultado desde http://www.laopinion. com.co/noticias/index.php?option $=\mathrm{com}_{-}$co ntent\&task $=$ view\&id $=413220 \&$ Itemid $=77$

Morin, Edgar. (1997). "La necesidad de un pensamiento complejo". Revista Universidad del Valle, 8.

Moser, Caroline. (1996). Confronting Crisis. A Comparative Study of Household Responses to Poverty and Vulnerability in Four Poor Urban Communities. Washington, DC.: The World Bank.
Narayan-Parker, D. (1999). Bonds and Bridges: Social Capital and Poverty: World Bank.

Neiva, Eduardo. (1993). El arraigo de la corrupción y la superficialidad de los medios. Chasqui, 45, 79 - 84. Consultado 20 abril de 2013. http://europa.sim.ucm.es/compludoc/ AA?articuloId $=593185$

Radio Nizcor. (2011). "Congresistas colombianos procesados por relación con el paramilitarismo" [Prensa online]. En El Emilio, internacionales. Consultado 27 de marzo de 2013. http://www.revistaelemilio.com.ar/2011/04/ congresista-colombianos-procesados-por-relacion-con-el-paramilitarismo/

Orozco, Jorge. (2010). "Hay 20 millones de pobres en Colombia". Consultado, 2013, desde:http://historico.elpais.com.co/paisonline/notas/Mayo012010/1pobreza.html

Portafolio.co (2012). Informalidad laboral en Colombia llega al 51.3\%. [Prensa online] Consultado 27 de marzo de 2013 desde, http://www.portafolio.co/economia/informalidad-laboral-colombia-llega-al-513

Portales, Luis, \& García, Consuelo. (2009). Capital social: conceptualización, enfoques y mediciones. ASCOLFA.

Putnam, Robert David. (1995). Robert D. Putnam. Bowling alone: America's declining social capital, Journal of Democracy, 6(1), 65-78.

Putnam, Robert David. (2001). Bowling alone: The collapse and revival of American community. New York: Simon \& Schuster.

Putnam, Robert David, Leonardi, Robert, \& Nanetti, Raffaella Y. (1994). Making Democracy Work: Civic Traditions in Modern Italy. New Jersey: Princeton University Press.

Rodríguez Raga, Juan Carlos, \& Seligson, Mitchell A. (2013). Barómetro de las Américas 2013. Cultura política de la democracia en Colombia y en las Américas, 2012: hacia la igualdad de oportunidades (pp. 289). Bogotá: USAID, Vanderbilt University, LAPOP.

Romer, Paul M. (1989). Human capital and
Junio - Diciembre 2013 ISSN 0122-820X PP: $43-60$ 
No. 2

Junio - Diciembre 2013 ISSN 0122-820X

PP: $43-60$

growth: theory and evidence. National Bureau of Economic research. Consultado 10 de septiembre de 2013, desde http://papers.ssrn. com/sol3/papers.cfm?abstract_id=227284

Rubio, Mauricio. (1997). Los costos de la violencia en Colombia (Vol. 97): Centro de Estudios sobre Desarrollo Económico.

Ryan E., Carlin. (2013). "Las violaciones del Estado de derecho alimentan la tolerancia a los sobornos en las Américas". En: Perspectivas (p, 1-11). USAID, Vanderbilt University, LAPOP, 88.

Sala-i-Martin, Xavier, \& Schwab, Klaus. (2011). The Global Competitiveness Report: 2011-2012.

Sudarsky, John. (1999). "Colombia's social capital: the national measurement with the BARCAS": National Planning Office.

Sudarsky, John. (2002). "El Capital Social en Colombia: principales hallazgos". En Reflexiones sobre la Investigación en Ciencias Sociales y Políticas, Memorias Seminario Octubre, 2002 (pp. 201 - 206). Bogotá: Universidad Nacional.

Sudarsky, John. (2007). La evolución del capital social en Colombia, 1997-2005. Bogotá: Fundación Antonio Restrepo Barco.

Todaro, Michael P. (1985). Economic development in the Third World (3a ed.). New York: Longman inc.

Woolcock, Michael. (1998). "Social capital and economic development: toward a theoretical synthesis and policy framework". Theory and Society, 27(2), 151-208.

Woolcock, Michael. (2000). "Managing risk, shocks, and opportunities in developing economies: the role of Social Capital". En G. Ranis (Ed.), Dimensions of Developmet. New Haven, Conn: Yale Center for International and Area Studies.
Woolcock, Michael, \& Narayan, Deepa. (2000). "Social capital: Implications for development theory, research, and policy". The world bank research observer, 15(2), 225-249. 